molecular device to promote ion flow across membranes.

Walter (Baas Becking Laboratory) reviewed the fossil evidence and Hayes the biogeuchemical evidence for early evolution. They concluded that life was present by 3.5 and photosynthesis by 2.8 Gyr ago in the Warrawoona and Fortescue strata of Western Australia.

An extended discussion followed about the importance of sulphur in living systems, the geological record of sedimentary sulphate and $\mathrm{S}$ isotopes, the possible importance of methanogens and the question of when free $\mathrm{O}_{2}$ from photosynthesis first became an important atmospheric gas. Groves noted that sulphates are uncommon in Archaean rocks whereas sulphides are abundant. Donnelly added that the sulphur isotope record of stratabound volcanogenic sulphide deposits calls for very low concentrations of oxidized sulphur in the Archaean hydrosphere. The record observed is consistent with the convergence of three important and perhaps related events $\sim 2$ Gyr ago: (1) the beginning of free $\mathrm{O}_{2}$ as a permanent component of the atmosphere, (2) the end of methanogenesis as an important function of the biomass, and (3) the onset of dissimilatory sulphate reduction.

The emergence of the eukaryotic cell at some time after $\sim 2$ Gyr ago was the focus of the final session at Jindabyne. Walter reviewed the biogeological evidence for the rise of $\mathrm{O}_{2}$ during Earth history, the probable time of emergence of the eukaryotes and alternative hypotheses for eukaryotic origins. Runnegar (New England University) observed that even anaerobic eukaryotes need $\mathrm{O}_{2}$ for the production of collagen and components of the cell wall. Thus eukaryotes probably made their first appearance later than $2 \mathrm{Gyr}$ ago. But how? Langridge observed that differences between organellar and nuclear DNA of eukaryotes could result either by derivation of the organellar DNA from the nucleus by a transfer analogous to that involved in the origin of some viruses, or by endosymbiosis.

Lastly the conference turned to the origin of membrane-bound organelles, considered distinctive of the eukaryotic cell. Langridge noted that cytochrome $c$ amino acid sequences and 5S rRNA nucleotide sequences imply a date of $\sim 1.8$ Gyr ago for the divergence of the eukaryotic from the prokaryotic level of organization - a date consistent with biogeological evidence. No serious alternative was offered to serial endosymbiosis for the origin of the eukaryotic complement of membranebound organelles. Discussion concentrated instead on which of these organelles might have come first. Starting with a nucleated host, most preferred first to add mitochondria of probable bacterial origin and then chloroplasts, whose 16S rRNA sequences imply a blue-green algal origin.

\section{Was Galileo 2,000 years too late?}

\section{from David $W$. Hughes}

SIDEREUS NUNCIUS was published in Venice in 1610 and revealed to the renaissance world "great, unusual, and remarkable spectacles . . . . . . as observed by Galileo Galilei, Gentleman of Florence, Professor of Mathematics in the University of Padua, with the aid of a spyglass"'. The title page also announced that the greatest discovery was that of the "four planets swiftly revolving about Jupiter at differing distances and periods, and known to noone before the Author recently perceived them and decided that they should be named The Medician Stars". One of Galileo's notebooks records how Jupiter was seen on 7 January 1610 with three companion 'fixed stars', two to the east and one to the west. Galileo concluded, after a week of observation, that "there are three wandering stars around Jupiter, previously invisible to everyone'".

Galileo's claim to priority has recently been shown to be unjustified; and it is not a matter of being just pipped at the post, but of some 2,000 years.

$\mathrm{Xi}$ Ze-zong, of the Institute for the History of Natural Sciences, Academia Sinica, has recently reported in Chinese Astronomy and Astrophysics (5, 242; 1981) that Gan De observed Ganymede in the summer of $365 \mathrm{BC}$.

Gan De was one of the earliest Chinese astronomers and was an assiduous observer of the heavens, and in particular of the planet Jupiter. He wrote two books, Treatise on Jupiter and Astrological Prognostications. Both have been lost but fortunately portions have been preserved in The Kaiyuan Treatise on Astrology which was compiled by Qutan Xida between AD 718 and 726. Gan De is quoted as having said:

"In the year of chan yan . . ., Jupiter was in Zi, it rose in the morning and went under in the evening together with the lunar mansions Xunü, $\mathrm{Xü}$ and Wei. It was very large and bright. Apparently, there was a small reddish star appended to its side. This is called 'an alliance'.",

The reference to the zodiacal division $\mathrm{Zi}$ and the lunar mansions reveals that the observation was made in the summer of 365 $\mathrm{BC}$ and the use of the term 'in alliance' that the small star is described as a subsidiary of Jupiter. The colour 'chi' is a light red.

$\mathrm{Xi}$ Ze-zong puts forward no explanation as to why this reference to a satellite of Jupiter has been overlooked until now.

Which satellite was it? Jupiter has four 'galilean' satellites, Io, Europa, Ganymede and Callisto, and when Jupiter is in opposition, at its closest to Earth, these have magnitudes of $4.9,5.3,4.6$ and 5.6 respectively (a change of 1.0 in magnitude is equivalent to a factor of 2.5 in brightness and brignt objects have low magnitude values). At opposition the maximum angular separations between the satellites and Jupiter are 2.3, 3.7, 5.9 and 10.1 arc min respectively. Ganymede is thus the brightest moon and can be as much as 5.9 arc min away from Jupiter - one-sixth the apparent diameter of the Moon. Unfortunately, Jupiter then has a magnitude of around -2.6 and is thus 760 times brighter.

$\mathrm{Xi}$ Ze-zong used a planetarium to simulate two sources differing in brightness by a factor of 760 and separated by 5.9 arc min. People with good eyesight could detect the fainter object. Xi Ze-zong could, however, have saved himself some trouble by turning to a more recent 'ancient tome'. Admiral William H. Smyth wrote in his Celestial Cycle, Vol. 1 (1844) that

"Certain esprits fort express surpise that Galileo should have been so gratified by this discovery since they hold that the satellites of Jupiter are often seen with the naked eye and they cite the Apennines and Etna and the West Indies and various other fine-climate places as the spots where such a feat is frequently done."

Smyth concluded, however, that the observer must not only be at a site of fine seeing and excellent atmospheric clarity but must also possess "visual organs of extraordinary power".

Two problems still exist. We cannot be sure whether Gan De saw Ganymede or Callisto. Ganymede is the more probable simply because it is the brighter. The reference to the 'light red' colour is also mystifying. Ganymede is too faint for its colour to be perceived with the naked eye. Even in a telescope it appears white and has a very similar colour to that of Jupiter.

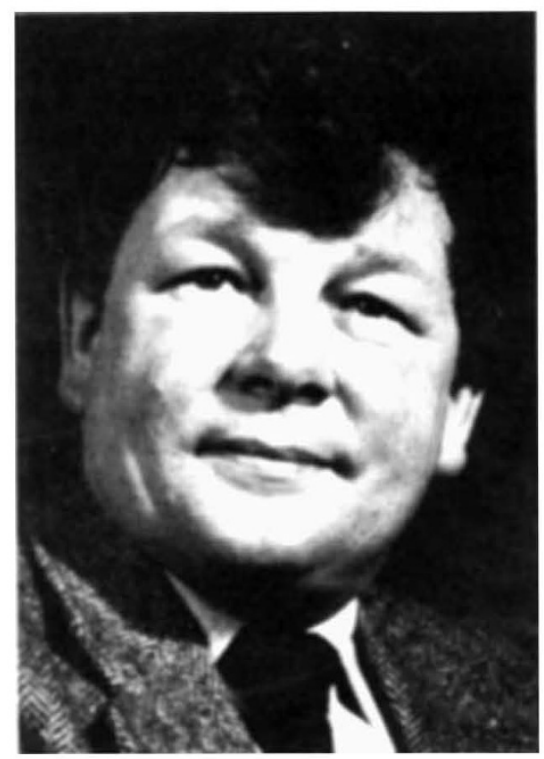

This the hundredth article written written for News and Views by David W. Hughes, a lecturer in physics and astronomy at the University of Sheffield. The first, "Meteors and Meteorology', was published on 13 June 1970 Nature 226, 1008). 